BULLETIN Bulletin hispanique

HISPANIQUE Université Michel de Montaigne Bordeaux

116-1 | 2014

Varia

\title{
La présence de Verhaeren, Rodenbach et Maeterlinck en Catalogne jusqu'en 1939
}

critiques, traducteurs, éditeurs et revues

Manuel Llanas et Ramon Pinyol

\section{OpenEdition}

\section{Journals}

Édition électronique

URL : http://journals.openedition.org/bulletinhispanique/3237

DOI : 10.4000/bulletinhispanique.3237

ISSN : $1775-3821$

Éditeur

Presses universitaires de Bordeaux

Édition imprimée

Date de publication : 1 juin 2014

Pagination : 349-361

ISBN : 978-2-86781-931-5

ISSN : 0007-4640

\section{Référence électronique}

Manuel Llanas et Ramon Pinyol, «La présence de Verhaeren, Rodenbach et Maeterlinck en Catalogne jusqu'en 1939 », Bulletin hispanique [En ligne], 116-1 | 2014, mis en ligne le 01 juin 2017, consulté le 26 mai 2020. URL : http://journals.openedition.org/bulletinhispanique/3237 ; DOI : https://doi.org/ 10.4000/bulletinhispanique.3237 


\title{
La présence de Verhaeren, Rodenbach et Maeterlinck en Catalogne jusqu'en 1939: critiques, traducteurs, éditeurs et revues
}

\author{
Manuel Llanas \& Ramon Pinyol \\ Université de Vic - Catalogne, Espagne
}

L'article passe en revue la réception, aussi bien en catalan quien espagnol, des trois écrivains belges en Catalogne. Alors quill faut attendre les débuts du XX' siècle pour que paraisse une traduction de Rodenbach, Verhaeren et Maeterlinck sont accueillis par le Modernisme catalan dès la fin du XIX siècle. Des trois auteurs, Maeterlinck est celui qui a laissé l'empreinte la plus profonde et la plus durable.

Mots clés: Verhaeren, Rodenbach, Maeterlinck, Catalogne.

El articulo hace un recorrido por la recepción de los tres escritores belgas en Cataluña, tanto en catalán como en español. Mientras que para la aparición de traducciones de Rodenbach hay que esperar a principios del siglo XX, Verhaeren y Maeterlinck son acogidos por el Modernismo catalán desde finales del siglo XIX. De los tres, Maeterlinck es quien deja un rastro más intenso y duradero.

Palabras claves: Verhaeren, Rodenbach, Maeterlinck, Cataluña.

This article reviews the reception in Catalonia of the three Belgian writers, both in Catalan and in Spanish. While translations of Rodenbach would not come out until the early $20^{\text {th }}$ century, Catalan Modernism greeted Verhaeren and Maeterlinck at the end of the $19^{\text {th }}$ century. Of the three, Maeterlinck left the most remarkable and lasting imprint.

Keywords: Verhaeren, Rodenbach, Maeterlinck, Catalonia. 


\section{INTRODUCTION}

Les premiers témoins documentaires de la présence de cette trinité littéraire francophone belge en Catalogne se situent dans les pages de deux revues du Modernisme catalan ${ }^{1}$, un mouvement esthético-culturel étroitement lié à l'Art nouveau et au Jugendstil, à cheval entre le $\mathrm{XIX}^{\mathrm{e}}$ et le $\mathrm{XX}^{\mathrm{e}}$ siècle. Il s'agit de L'Avenç (1881-1884 et 1889-1893) et de la revue qui lui succède, Catalònia (1898 et 1899-1900), qui furent les porte-paroles d'un noyau intellectuel qui, le regard tourné vers le nord, aspirait à européaniser la littérature catalane et, donc, à la mettre au niveau de celles qui donnaient le ton de la modernité culturelle. Ce désir est synthétisé dans une affirmation lapidaire que Joan Maragall écrivait en 1893, en français, dans une lettre à un de ses amis : "Et c'est toujours du Nord que nous vient la lumière ». Dans la même lettre, Maragall mettait en garde son correspondant contre le fait que, dans les modes littéraires barcelonaises les plus en vogue, il ne faut pas croire " que Zola est encore le patron de tout... Non, mon fils, non; Ibsen, Tolstoï, Maeterlinck, Nietzsche ». Etroitement lié au symbolisme on trouve la littérature de deux écrivains belges, Verhaeren et Maeterlinck, ce dernier passant, tout au moins dans les milieux barcelonais, comme appartenant aux toutes dernières modes européennes. Quant à Rodenbach, il commença à être connu à Paris parmi les intellectuels catalans qui y résidaient et, au début, grâce exclusivement au roman Bruges-la-Morte. En définitive, et selon tous les témoins et indices, les trois écrivains entrent pour la première fois dans les littératures hispaniques à travers Barcelone et, par conséquent, à travers la littérature catalane. À partir de ce moment initial, leur présence en Catalogne jusqu’en 1939 sera très inégale - tel que nous l'expliquerons.

\section{ÉMILE VERHAEREN ET LA RÉACTION CONTRE L'INDUSTRIALISME}

La première trace qu'on puisse détecter d'Émile Verhaeren se trouve dans un numéro de la revue L'Avenç, qui reproduit un poème en langue originale (" La Ville ", extrait du livre Les Campagnes Hallucinées, qui venait d'être publié à Bruxelles), précédé d'une présentation détaillée de l'écrivain et du texte choisi. Une présentation anonyme qui contient des informations précises et à jour et qui manifeste une vision critique, juste et aiguë, des ressorts éthiques et esthétiques du poète. Les lignes suivantes illustrent ce propos:

1. En l'absence d'une étude exhaustive, on peut cependant observer que la connaissance de Maeterlinck en Catalogne s'est opérée par diverses voies. En marge du mouvement moderniste, se situe le poète le plus remarquable de son temps, Jacint Verdaguer qui dans un article daté de 1900 concernant le mystique belge Ruysbroek signale que seuls le connaissent " quelques dévots de Maeterlinck» (El Pensament Català, no 4 (1900), p. 109-111; reproduit dans Jacint Verdaguer, Prosa, Barcelona: Proa, 2003, p. 460). 
Émile Verhaeren est un des jeunes poètes belges contemporains au talent des plus original et qui s'est forgé un nom grâce à sa forme libre de comprendre la versification et la poésie. [...].

Les Campagnes Hallucinées, Les Villes Tentaculaires et Les Aubes formeront une trilogie. Dans Les Campagnes Hallucinées, la ville, peut-être Londres, est décrite comme un énorme poulpe qui étend ses tentacules partout (voies, routes, télégraphes, etc.), absorbant avec elles toute vie émanant des campagnes. Ces dernières apparaissent déjà comme des lieux enchantés pleins d'illuminés et de fous: la vie saine a disparu, ne laissant que des fantômes fuyants, extravagants, malades et criminels de ce qu'un jour furent les paysans, les villages, les paroisses, etc. On copie le premier fragment, intitulé La Ville, du volume Les Campagnes Hallucinées, car il est le plus facile à comprendre et parce qu'il forme à lui seul un petit poème complet et indépendant. En plus, dans ce fragment, l'un des meilleurs du volume, on peut comprendre Verhaeren, le poète à l'imagination victorhuguesque, dont la force de peinture et de compréhension et synthèse s'apparente à celles de Zola.

Dans le numéro 2 de la revue Catalònia du 10 mars 1898, Joan PérezJorba consacrait au poète un article qui, sous le titre "Emili Verhaeren ", constituait toute une étude critique et faisait un bilan et une synthèse des clés de sa littérature: objectifs, émotions, idéologie, contexte national, évolution et situation générationnelle. Mais plus que tout, le critique soulignait, avec des accents empreints de lyrisme, les liens profonds qui unissaient l'écrivain à son pays natal. En même temps, il le situait dans la lignée de la "hardie et nombreuse jeunesse qui dirige et représente le mouvement actuel» des lettres belges (aux côtés de, par exemple, Maeterlinck, Lemonnier, Eeckhoud, Arnould, Van Lerberghe ou Rodenbach), et il affirmait, pensant à la Catalogne:

Il est intéressant d'observer ces réveils individuels des nationalités; leur triomphe politique indique, pour l'avenir, la préparation d'une harmonie universelle au sein des peuples: exalter l'individualité afin d'obtenir la sociabilité. Toutefois, nous sommes encore tout juste dans une période de sommeil au réveil et à l'individualisation.

Un exemple remarquable de l'accueil fait à Verhaeren est celui de son livre España negra - centré, cependant, sur le Pays Basque et la Navarre -, traduit en espagnol et illustré par l'important peintre espagnol Darío de Regoyos. Il commença à paraitre au cours du mois de décembre 1898 dans la revue barcelonaise $L u z$ - écrite en espagnol et en catalan - et quelques mois plus tard l'ouvrage fut publié en format de livre à Barcelone également. Comme c'était prévisible, il provoqua des réactions diverses, allant du rejet pour avoir dépeint une Espagne qui résultait offensante voire pleine de clichés jusqu'à l'adhésion plus ou moins inconditionnelle pour dénoncer les tares du caractère espagnol.

En marge de ces revues, dans l'anthologie Trasplantades. Poesies franceses contemporànies, publiée en 1910, on trouve un poème de Verhaeren, "Novembre ». Un poème que commente, dans le prologue, une interprétation condensée de la littérature de Verhaeren introduite par les mots suivants : "Verhaeren se présente comme un tempérament synthétiseur du caractère essentiel du pays où il est né. La Flandre au complet passe par ses vers». 
La présence de Verhaeren ira s'amenuisant une fois l'étape moderniste passée. Toutefois, pendant la Grande Guerre, au cours de laquelle le poète s'engage activement dans la défense de son pays, la presse barcelonaise donne souvent des nouvelles de ses activités en Europe et de sa nouvelle production, ainsi que de quelques conférences concernant son œuvre ou de concerts donnés à Barcelone où certaines de ses pièces sont mises en musique. De même, sa mort, survenue dans des circonstances tragiques le 26 octobre 1916, à Rouen, à l'issue d'une conférence, est objet d'attention des médias barcelonais. En conséquence, plusieurs critiques littéraires lui consacrent des articles nécrologiques.

Entre 1915 et 1917, une revue barcelonaise très clairement francophile, Iberia, traduit en espagnol quatre textes en prose et un poème de Verhaeren, tous destinés à souligner l'effort de guerre et la souffrance endurés par la Belgique et à blâmer en même temps l'Allemagne. Néanmoins la publication la plus favorable à la figure civique et littéraire de Verhaeren est Estudio, porte-parole d'une Société d'Etudes économiques implantée à Barcelone qui, très sensible à la guerre en Europe, traduit entre 1914 et 1920, neuf poèmes de l'écrivain et lui consacre deux articles qui font l'éloge de son idéologie et de son esthétique. Finalement, il faut citer la publication de la pièce théâtrale Le Cloître dans la collection La Novel.la Teatral Catalana, qui en 1919 a sans aucun doute voulu profiter de l'écho du succès obtenu par sa représentation au théâtre Romea, de Barcelone, en mai.

\section{Georges Rodenbach eT LeS VILles DéCAdentes}

Vers la fin du XIX ${ }^{\mathrm{e}}$ siècle, et pour les militants de l'esthétique décadentiste européenne, Venise et Bruges deviennent deux endroits symboliques, deux villes mythiques qui, situées en dehors du temps, survivaient en ruines et en pleine décadence. D’où le surnom de villes mortes qui leur est octroyé parce qu'elles n'avaient pas su préserver la splendeur du passé. Ce mythe, exploité à fond par la littérature et la peinture, dans le cas de Bruges semble remonter à Busken Huet, un agitateur politique néerlandais, qui, après avoir visité la ville en 1879, la baptisa " Bruges, la ville morte ». L'expression "villes mortes » fit fortune et fut encore consolidée par un roman de Georges Rodenbach, Bruges-la-Morte (1892), dans lequel le protagoniste, déprimé par une tragédie familiale, évoque, seul au milieu de la ville, un monde de suggestions, de mélancolies et de rêves. Le succès du roman de Rodenbach se projette d'abord dans la littérature elle-même (Mauclair, Mallarmé, Barrès, Huysmans) ; Bruges entre pleinement dans la thématique littéraire décadentiste, tout en offrant un décor urbain approprié au spleen, au rêve et à l'angoisse qui se réfléchissent dans les pierres, l'humidité, les eaux noirâtres et mortes des canaux, dans les sons des cloches, le pas tranquille des béguines, dans la froidure, dans le silence. La ville exerce une sorte de charme qui la déréalise et la transforme en motif d'exploration des espaces intérieurs inconnus. 
Forts de ce référent européen, au début du XXe siècle, un groupe d'écrivains modernistes catalans faisait de la ville de Gérone - située à peu près à $80 \mathrm{~km}$ au nord de Barcelone - un objet littéraire, comme Rodenbach l'avait fait avec Bruges. Dans ce parallèle, les deux villes partageaient la même atmosphère et un même paysage fait de grisaille, d'humidité, de brouillard, de silence, de jardins abandonnés ou fermés par des murs de pierre couverts de lierre et l'omniprésence de la religion (clochers, prêtres, religieuses et béguines). Cependant, l'expérience littéraire catalane n'a pas seulement pour but d'évoquer sur un ton nostalgique le passé ou de chercher des espaces propices au rêve, mais plutôt exposer la critique d'une société immobile qui veut se régénérer et, donc, lutter contre le provincialisme culturel et social. L'entreprise est menée par un groupe rebelle et innovateur qui prend le thème de la ville morte comme prétexte pour lutter contre le provincialisme culturel, pour manifester son opposition à la ville ancrée dans le passé, inactive et inerte. Qui en faisait partie? Des intellectuels allant de Xavier Montsalvatge à Carles Rahola et Rafael Masó, de Prudenci Bertrana à Miquel de Palol, de Santiago Rusiñol à Eugeni d'Ors et Josep Carner. Selon la professeure Margarida Casacuberta, tous «s'attachent à présenter Gérone sous une double dimension, celle de miroir de l'âme et celle d'espace de réflexion sur le moi, et de la ville cléricale, provinciale, ancrée dans le passé et susceptible de faire partie de l' "Espagne noire " qu'aussi bien Carner que Rusiñol, ou les écrivains contemporains de Gérone s'étaient proposé de régénérer à travers la littérature, l'art et la culture en général».

Carner est une des figures les plus remarquables de la littérature catalane du XX $X^{e}$ siècle, qui - dans un article publié dans un journal qui fait partie d'une série et sous le pseudonyme d'Oleguer Recó («Coses gironines. Evocació mortuòria» ["Choses de Gérone. Evocation mortuaire »]. La Veu de Catalunya, 17-XI-1906) - dessine pour la première fois les traits essentiels qui associent Gérone à Venise et Bruges: traversée par quatre rivières à l' apparence de canaux aux eaux calmes, dominée par la cathédrale, comptant de nombreuses églises et des couvents, froide et humide, elle semble, somme toute, plus nordique que méditerranéenne. Ce texte de Carner reflète avec beaucoup de fidélité la description de Bruges faite par Rodenbach : obsessions morbides qui se dégagent du décor urbain, espace atemporel, silence compact, omniprésence de la mort, arrière-goût nordique d'obscurité, d'humidité et de froidure. Dans trois sonnets de la même année 1906, intitulés conjointement Gerunda, Carner parfait encore cette image de la ville et établit un cliché qui, assumé de forme consciente, se trouve dans d'autres créations littéraires d'une importance (tels que Josafat et El vagabund [Le vagabond], de Prudenci Bertrana, où sont mis en contraste le vice, la violence, la mort et des éléments religieux et mystiques).

Dans le même volume de traductions intitulé Trasplantades. Poesies franceses contemporànies qui a déjà été mentionné, est reproduit un poème, «Els ulls de les dones " ["Les yeux des femmes »], de Rodenbach, qualifié par Pérez-Jorba dans son prologue de «tendre amateur du brouillard», «dont les vers informes ne correspondaient pas à la mélodie émotive qui les inspirait». 
En 1930 parut une anthologie poétique de Rodenbach, traduite en espagnol et précédée par une présentation biographique et esthétique détaillée, rédigée par les deux traducteurs (Luis Guarner et Miguel Alejandro Rives). Ils comparent l'écrivain à Verhaeren et Maeterlinck («la trinité suprême de la lyrique belge moderne») qui, tout en le caractérisant, semblent avoir été contaminés par le fragile lyrisme automnal des poèmes choisis:

Si les sommeillantes villes flamandes, accablées par le poids de leur passé glorieux et leur mélancolie actuelle, ont une âme et un langage qui arrive aux sens humains, c'est sans doute l'âme subtile qui anime la poésie de Rodenbach, et la voix mélancolique de ses vers, l'ineffable idiome de ce pathétique mystère.

Rodenbach est le poète exquis du Silence - «musicien du silence et peintre de la solitude» - qui sait ausculter la vie intérieure des choses [...].

\section{Maurice Maeterlinck ou la poÉsie de la suggestion}

Contrairement à Verhaeren et à Rodenbach, Maeterlinck est nettement plus présent au sein des lettres hispaniques et son œuvre largement plus étudiée, surtout dans son versant théâtral. Actuellement il semble que tout le monde accepte que ce fut Santiago Rusiñol qui eut le premier contact - à Paris entre 1888 et 1892 - avec l'œuvre littéraire de Maeterlinck. On ne peut cependant pas totalement exclure le fait qu'il ait été également connu - en français - à travers des références faites dans des publications périodiques et grâce à la présence d'un fonds de ses ouvres originales dans les librairies barcelonaises, circonstance que certains critiques ont contextualisée dans la "préséance de Barcelone sur Madrid » à la fin du XIX siècle (Vauthier, 2001: 109-112 i 118). D'un point de vue documentaire, c'est toutefois le critique Joan Sardà - un des théoriciens les plus solides du naturalisme littéraire - qui nous livre le premier texte sur l'écrivain belge.

Suite à la traduction par Pompeu Fabra de L'Intruse en 1893 et Interior en 1898 puis de sa représentation à Sitges - épisodes largement divulgués par divers critiques (Pérez de la Dehesa, 1971: 577; Litvak, 1968: 186-189; Vauthier, 2001: 119-123, 126-128 i 131-134) - plusieurs articles intéressants sont publiés et quelques dramaturges se proclament fidèles disciples de Maeterlinck. A remarquer tout particulièrement Adrià Gual qui incorpore dans sa production théâtrale une série d'innovations (telle que la technique du dialogue et du silence) empruntées à l'auteur belge (Litvak, 1968: 193; Salaün, 2001: 232 et 235; Vauthier, 2001: 135-137). Malgré cela, l'enthousiasme n'est pas unanimement partagé. Parmi ceux qui different ou se montrent réticents à l'esthétique de Maeterlinck, il y a quelques intellectuels de premier plan comme Josep Yxart, Joan Maragall (Litvak, 1968: 190-192; Salaün, 2001: 224; Vauthier, 2001: 134-135) ou Jaume Brossa, qui en 1898 qualifiait Barcelone de «patrie adoptive de Maeterlinck». Les tournées de la compagnie théâtrale du dramaturge contribuent à la diffusion de l'œuvre dramatique de Maeterlinck en Espagne. À sa tête Georgette Leblanc, femme de l'écrivain, qui visita Madrid et Barcelone la première fois en 1904 (Salaün, 2001: 226-227). 
À partir des années 20, l'intérêt pour le théâtre de Materlinck se poursuit avec quelques oscillations; désormais sa poésie et ses romans suscitent eux aussi un intérêt, preuve en est, dans le domaine de la poésie, le volume de traductions Trasplantades. Poesies franceses contemporànies, déjà mentionné à propos de Verhaeren et Rodenbach; Maeterlinck y est représenté par une «Cançó» qui commence ainsi "J'ai cherché trente années, mes sœurs» tandis que, dans l'étude préliminaire, Joan Pérez-Jorba s'efforce de l'inscrire dans le courant symboliste.

En ce qui concerne la narration, les trois traductions immédiatement postérieures à 1911 - année de la concession du prix Nobel de littérature à l'écrivain - cherchent sans aucun doute à se servir de l'écho suscité par l'évènement. Mais, comme on peut le constater dans l'annexe, au cours des années 20 et 30, Maeterlinck a continué à être un auteur traduit, tant en catalan qu'en espagnol, et assez fréquemment présent sur les affiches théâtrales barcelonaises, bien que quelques unes des pièces représentées n'ont pas eu la chance d'être éditées par la suite. Dans la période qui nous occupe, en plus des articles le concernant et son ouvre, recueillis dans l'annexe, et en guise de témoignage anecdotique de l'intérêt qu'il éveillait, on peut rappeler qu'il se faisait des représentations et des lectures commentées de ses œuvres dans les associations ouvrières, qu'on suivait les vicissitudes de ses premières en Europe (un article en première page de La Vanguardia du 1 octobre 1903, intitulé «Maeterlinck, silbado en Bruselas», rapporte l'échec de la première de El milagro de san Antonio) et qu'on annonçait même son divorce d'avec l'actrice et chanteuse Georgette Leblanc en août 1919 : grande propagandiste de son œuvre, elle s'était rendue à Barcelone à plusieurs reprises pour y représenter des pièces de Maeterlinck.

\section{Conclusions}

1. Maeterlinck a eu une influence décisive sur le groupe moderniste pendant la décade de 1890. En outre, des trois écrivains pris en considération, il est celui qui laisse-de loin-l'empreinte la plus profonde et durable. Ce fait est confirmé comme donnée la plus représentative, par les 23 traductions répertoriées - 14 en catalan et 9 en espagnol - : certaines d'entre elles ne se publient pas mais se font connaître à travers des représentations théâtrales. Maeterlinck a joui d'une grande renommée, non seulement à cause du prix Nobel de littérature qui lui a été concédé en 1911 mais aussi à cause de quelques-unes de ses œuvres mises en musique par des compositeurs célèbres.

2. Verhaeren est présent à travers la poésie, le théâtre et des chroniques intégrées dans le volume intitulé España negra, qu'il signe avec le peintre Regoyos et qui se publient traduites pour la première fois en 1898 dans une revue et l'année suivante en forme de volume, dans les deux cas à Barcelone. Quant à sa mort tragique, survenue pendant la Grande Guerre, la presse barcelonaise s'en fit largement écho, notamment la presse de sensibilité francophile. 
3. Comparativement, Rodenbach est l'écrivain qui a eu le moins de rayonnement, en partie à cause de sa mort prématurée. Pourtant, son roman sur Bruges, lu uniquement en français pendant de nombreuses années, devint la référence obligée du mythe des villes mortes. Un mythe fécond et rentable qui nourrit la vision littéraire de Gérone, une ville catalane au profil semblable à celui de Bruges. Des écrivains catalans postérieurs (Josep M. de Sagarra, Josep Pla, Salvador Espriu, Joan Perucho) et d'autres plus récents (Narcís Comadira) en subissent encore l'influence.

4. Un éventail très hétérogène de traducteurs et de critiques rassemble tout aussi bien des intellectuels de haute volée (avec à leur tête Pompeu Fabra) que des personnalités moins connues. L'œuvre de Maeterlinck est à nouveau dans ce milieu la plus commentée, depuis la fin du XIX ${ }^{\mathrm{e}}$ siècle jusqu'aux années $30 \mathrm{du} \mathrm{XX}^{\mathrm{e}}$ siècle et ce, par différentes générations de critiques, qui, quand ils s'en distancient (c'est le cas de Joan Sardà, Josep Yxart ou Jaume Brossa, partisans d'une esthétique plus proche du naturalisme) ne manquent pas de lui manifester un grand respect.

\section{Bibliographie}

Anoll Lídia, Pel-leds i Melisanda de Maurice Maeterlinck. Introducció, lectura i traducció, Lérida, Universitat de Lleida; Pagès Editors, 1999, 176 p.

Castellanos Jordi, Literatura, vides, ciutats, Barcelone, Edicions 62, 1997, 193 p.

Casacuberta Margarida, "Un segle en imatges. Girona, pedra viva ", L’Avenç, juin 2001, no 259, p. 80-83.

Gual Adrià, Mitja vida de teatre. Memòries, Barcelone, Aedos, 1960, 356 p.

Litvak Lily, " Maeterlinck en Cataluña ", Revue des Langues Vivantes, 1968, vol. XXXIV, p. 184-198.

Maragall Joan, Obres completes, vol. I, Barcelone, Selecta, 1960, 1.329 p.

Palau de Nemes Graciela, "La importancia de Maeterlinck en un momento crítico de las letras hispanas ", Revue Belge de Philologie et d'Histoire, 1962, no 40, p. 714-728.

Pérez de la Dehesa Rafael, "Maeterlinck en España ", Cuadernos Hispanoamericanos, 1971, no 255, p. 572-581.

Salaün Serge, "Maeterlinck en Espagne ", dans Aymes J. R. et Salaün S. (sous la dir. de), Le métissage culturel en Espagne, Paris, Presses de la Sorbonne Nouvelle, 2001, p. 221-241.

Senabre Ricardo, "De nuevo Maeterlinck en España (Testimonios y documentos) », Correspondance, 2000, no 6, p. 136-148.

Vauthier Bénédicte, "Réception et influence du premier théâtre de Maeterlinck: de Barcelone à Madrid ", Annales de la Fondation Maurice Maeterlinck, XXXII, 2001, p. 101-154.

Vauthier Bénédicte, «Le premier théâtre de Maeterlinck en Espagne: Retour sur une reception bicéphale», Textyles, no 41 2011, p. 99-114. 


\section{Annexe}

\section{LiSTE DE TRADUCTIONS ET TEXTES CRITIQUES}

Dans cette annexe, nous dressons une liste ordonnée chronologiquement des témoignages de la présence dans des livres et des revues catalanes des trois écrivains belges jusqu'en 1939. Y figurent sans aucun tri des textes en langue originelle, des traductions en catalan et en espagnol et des articles concernant la figure et l'œuvre de chacun des trois auteurs. Les revues citées sont toujours issues de la ville de Barcelone si on n'indique pas autrement.

\section{Émile Verhaeren}

«La Ville» [texte en langue originale, accompagné d'une présentation], L'Avenç, (31 octobre-15 novembre 1893), [2 époque], V, no 20-21, p. 322-323.

Pérez Jorba Joan, «Emili Verhaeren», Catalònia, no 2 (10-3-1898), p. 19-25.

España negra, traduction et illustrations de Darío de Regoyos, Luz, décembre 1898, $n^{\circ} 8-12$, p. 93-95, 100-103, 116-117, 129-131 et 135-140. [La revue cessa de paraître et la publication de l'œuvre de Verhaeren s'interrompit au $3^{\mathrm{e}}$ chapitre (l'œuvre a six chapitres et une conclusion)]

España negra, présentation de Rodrigo Soriano, traduction et illustrations de Darío de Regoyos. Barcelone, Impremta de Pedro Ortega, 1899. [En fait, il s'agit d'une traduction de fragments des articles originaux de Verhaeren publiés dans L'Art Moderne de Bruxelles après le voyage (1888), combinés avec des commentaires et narrations de Regoyos, qui illustre aussi le livre avec 27 gravures et 7 originaux au buis].

«Novembre» (poème), traduction d'Emili Guanyavents, Joventut, (19-11-1903), $\mathrm{n}^{\circ} 197$, p. 760.

«Novembre», dans Trasplantades. Poesies franceses contemporànies, traduction d'E. Guanyavents et prologue-étude de J. Pérez Jorba, Barcelone, L’Avenç, 1910, p. 101-103. (Commentaire de la poésie de Verhaeren à la p. XLI).

"Muerte cristiana», dans Poesías excelsas (breves) de los grandes poetas, traducidas directamente en verso de sus idiomas respectivos por Fernando Maristany [Traduits directement en vers de leurs langues respectives par Fernando Maristany], Barcelone, A. López, 1914, p. 157-160.

«Pròleg» à Paul Spaak, Kaatje (Caterina), quatre actes en vers traduits en catalan par Frederic Pujulà, Barcelone, Impremta Artís, 1914. [Note au pied de la première page du prologue: «Conferència llegida a Brussel.les el dia de la 50a representació de Kaatje per son autor, l'Emili Verhaeren - qui amablement ens ha concedit el permís de traduir-la i encapçalar amb ella aquesta edició -, i a Barcelona el dia de l'estrena per en Frederic Barceló». [Conférence lue à Bruxelles le jour de la $50^{\mathrm{e}}$ représentation de Kaatje par son auteur, Emile Verhaeren - qui nous a aimablement concédé la permission de la traduire et la mettre en tête de cette édition]

«Lírica belga. Émile Verhaeren». «La noche», «¿Sé dónde?» i «El último sol», traduction de Matilde Ras, Estudio, juillet 1914, no 19, p. 99-102.

«La Alemania incivil» [traduction anonyme d'un article paru dans Les Annales], Iberia, $17-4-1915, n^{\circ} 2$, p. 4. 
"La Bélgica sangrienta. "Villages de Flandre" " [traduction anonyme d'un fragment de l'œuvre citée], Iberia, 12-6-1915, no 10, p. 14.

F. de A. R, commentaire d' «El espíritu belga», d’Émile Verhaeren [article paru dans le no 11 de la revue La Renaissance (26 juin 1915)], Estudio, août 1915, no 32, p. 248-251.

Plana Alexandre, "Las ideas y el libro: Emilio Verhaeren, "La Belgique sanglante" " $L a$ Vanguardia, 3-9-1915.

«Un capítulo de Verhaeren. El alma moderna» [traduction anonyme du dernier chapitre de La Belgique sanglante], Iberia, 25-9-1915, no 25, p. 8-9.

F. de A. R. commentaire d'Émile Verhaeren, La Belgique sanglante (Paris, Nouvelle Revue Française, 1915), Estudio, décembre 1915, no 36, p. 478-480.

Díez Canedo Enrique, «La obra de Emilio Verhaeren y su visión de España», Estudio, avril 1916, no 40, p. 1-18.

«La tenacidad de Bélgica» [ni la provenance ni le traducteur ne sont indiqués], Iberia, 8-7-1916, no 66, p. 3-4.

Escofet José, «Emilio Verhaeren», La Vanguardia, 3-12-1916.

Plana Alexandre, «Las ideas y el libro: Verhaeren», La Vanguardia, 6-12-1916.

Massaguer Enrique, «Emilio Verhaeren», Estudio, janvier 1917, no 49, p. 34-44. [Il s’agit de la nécrologie, avec une valorisation esthétique et idéologique de l'œuvre]

«Lírica belga. Enrique [sic] Verhaeren». «La labradora», traduction de M.[atilde] R.[as], Estudio, mars 1917, no 51, p. 407.

«Lírica belga. Emilio Verhaeren». «El zuequero», traduction de M.[atilde] R.[as], Estudio, septembre 1917, no 57, p. 415.

«Un duelo» [poème], traduction de J. Socias Aldape, Iberia, 29-9-1917, p. 13.

"Lírica belga. Emilio Verhaeren». "Los huéspedes de diciembre», traduction de M. Ras, Estudio, décembre 1918, no 72, p. 393.

El claustre [Le Cloître], oeuvre en 4 actes, première à Barcelone le 2 mai 1919 au Teatre Català Romea, traduction de Carles Capdevila, Barcelone, Impremta Ràfols, (La Novel.la Teatral Catalana, II ${ }^{e}$ année, no 8), 1919. Compte-rendu de Lleal dans Catalana, no 55 (18-5-1919), p. 392.

«Lírica belga. Emilio Verhaeren». «Los monjes», traduction de M. Ras, Estudio, août 1919, no 80, p. 246.

"Los monjes», "Hacia el futuro» et «El esfuerzo», traduction de Fernando Maristany, dans Las cien mejores poesías (líricas) de la lengua francesa, Valencia; Buenos Aires, Cervantes, s. a. [entre 1916 et 1919], p. 183-188.

"Capvespre religiós», traduction de Miquel Ferrà, dans: M. Ferrà, Les muses amigues. XVI traduccions en vers, Sóller [Mallorca], Estampa de Marquès i Mayol, 1920, p. 27-28.

«Lírica belga. Emilio Verhaeren». «En el Norte», traduction de M.[atilde] R.[as], Estudio, janvier-février 1920, no 85-86, p. 59.

«Lírica belga. Emilio Verhaeren». «Las ciudades», traduction de J. P. Rivas, Estudio, mai 1920, no 89, p. 252-255.

«La nit», traduction de Joan Pons i Marquès, La Nostra Terra (Palma de Majorque), juin 1931, année IV, no 42, p. 227.

[Poème ou poèmes non identifiés dans une anthologie non localisée] Cien poemas escogidos. Recitaciones de Gloria Bayardo, Barcelona, Maucci, 1931. 


\section{GEORGES RoDENBACH}

[Poesía lírica], traduction de Luis Guarner et Miguel Alejandro Rives, Barcelone, Cervantes, (Las mejores poesías [líricas] de los mejores poetas, 57), 1930.

«Els ulls de les dones» et «Ânimes fosques» (poèmes), traduction d'Emili Guanyavents. Suplement a "De Tots Colors», no 33 (20-8-1910), p. IV-V.

«Els ulls de les dones», dans Trasplantades. Poesies franceses contemporànies., traduction d'E. Guanyavents et prologue-étude de J. Pérez Jorba, Barcelone, L’Avenç, 1910, p. 85-86. (Commentaire de la poésie de Rodenbach à la p. XXXVIII).

"El cofre» et «En provincia», traduction de Fernando Maristany, dans Las cien mejores poesías (líricas) de la lengua francesa, Valencia; Buenos Aires, Cervantes, s. a. [entre 1916 et 1919], p. 181-182.

Andrenio [pseudonyme d'Eduardo Gómez de Baquero], «A propósito de "Brujas, la muerta" ", La Vanguardia, 18-1-1919.

«Festeig» (narration), traduction d'Alfred Gallart et illustrations de Josep M. Castellar. D’Ací i D’Allà, février 1924, vol. XIII, no 74, p. 87-91.

«L'orgull» (narration), traduction d'Alfred Gallart et dessins de Joaquim Ros, D'Ací $i$ D'Allà, septembre 1923, vol. XII, no 69, p. 689-692.

\section{Maurice Maeterlinck}

Sardà Joan, «Los dramas de Mauricio Maeterlinck», La Vanguardia, 20-12-1892.

L'intrusa [L'intruse], traduction de Pompeu Fabra, L’Avenç, (15-31 août 1893) [2 $2^{\mathrm{e} e ́ p o q u e], ~ V, ~ n o ~ 15-16, ~ p . ~ 225-240 . ~}$

Cortada Alexandre, "Maurici Maeterlinck i el modern simbolisme franco-belga», L’Avenç, (15-31 août 1893) [2 époque], V, no 15-16, p. 243-248.

Casellas Raimon, «La Intrusa», La Vanguardia, 8-9-1893.

L'Intrusa, traduction de P. Fabra, Barcelone, L'Avenç, 1893. [C'est la traduction, déjà mentionnée, publiée dans la revue la même année]

Sardà Joan, «El último drama de Maeterlinck», La Vanguardia, 9-12-1896.

«Interior» [ Intérieur»] (théâtre) Traduction de Pompeu Fabra. Catalònia, 1 ère époque, $\mathrm{n}^{\circ} 2$ (10-3-1898), p. 25-33.

Brossa Joan, "A propòsit de "Pelleas i Melisanda"», Catalònia, 15 octobre 1898, no 16, p. 237-241.

Zanné Jeroni. «Lo tràgic quotidià segons en Maeterlinck», Joventut, 22-8-1901, no 80, p. 565-569. [Il s'agit d'un commentaire du Trésor des Humbles, «evangeli moral i estètic del gran autor belga» («évangile moral et esthétique du grand auteur belge»)]

Vilaregut Salvador, «De com en Maeterlinck és viu», Joventut, (18-9-1902), année III, no 136 p. 614-615.

"Cançons», Catalunya, 15 août de 1903, no 15, p. CIII, sans nom de traducteur. [on $\mathrm{y}$ trouve seulement un poème, de cinq strophes. Le premier vers de la première strophe est «Havia arribat al palau»]

«Quinze cançons» [Quinze chansons], traduction de Magí Sandiumenge, Catalunya, juin 1904, no 30, p. XXI-XXXVI.

Les quinze cançons de Maurici Materlinck, traduction de Magí Sandiumenge, s/l, s/d [1904]. [Couverture avec un dessin d'Octavi de Romeu. Il s'agit d'un tirage à 
part de la traduction parue dans Catalunya. Compte-rendu de Rafael Marquina dans Catalunya Artística, (26-1-1905), no 27, p. 67-68.]

«Prólogo» à Ralph W. Emerson, Siete ensayos, vol. 1, traduction de Pedro Umbert, Barcelone, Impr. Enrich y Ca, Biblioteca Sociológica Internacional, 1904.

La trilogía de la muerte: La Intrusa. Los ciegos [Les aveugles]. Interior, versión castellana de Antonio de Vilasalba, Barcelone, Antonio López, (Teatro Antiguo y Moderno, XVII) 1904. (Réédition: 1909).

"Cançó», dans Trasplantades. Poesies franceses contemporànies, traduction d'E. Guanyavents et prologue-étude de J. Pérez Jorba, Barcelone, L'Avenç, 1910, p. 50. (Comentaire de la poésie de Maeterlinck aux p. XXXVII-XXXVIII).

Andrenio [pseudonyme d'Eduardo Gómez de Baquero], «Maeterlinck», La Vanguardia, 1-4-1911.

«Los jardines de nuestras ciudades», La Vanguardia, 27-7-1911.

Les set princeses [Les sept princesses]. Sor Beatriu (Miracle en tres actes) [Soeur Béatrice], traduction de Josep Massó-Ventós, Barcelone, L’Avenç, (Biblioteca Popular de l'Avenç, 125), 1912.

La vida de las abejas [La vie des abeilles], traduction de Pedro de Tornamira, Barcelone, Montaner y Simón, 1913.

La inteligencia de las flores [L'intelligence des fleurs], traduction de Juan Bautista Enseñat, Barcelone, Montaner y Simón, 1914.

Díez-Canedo, Enrique. «La obra de Emilio Verhaeren y su visión de España». Estudio, $I^{\mathrm{e}}$ année, tome XIV, no 40 (avril 1916), 1-18.

L'arcalde de Stilmonde [Le bourgmestre de Stilmonde], "Drama en tres actes del poeta belga Maurici Maeterlinck. Estrenat al Gran Teatre Espanyol, de Barcelona, el 7 de març de 1919» [Drame en trois actes du poète belge Maurice Maeterlinck. Première à Barcelone au Gran Teatre Espanyol le 7 mars 1919], traduction de Salvador Vilaregut, La Escena Catalana, 24-4-1920, III ${ }^{\mathrm{e}}$ année (2 époque), no 55.

Monna Vanna, «Drama en tres actes de Maurici Maeterlinck estrenat al teatre Cirera de Barcelona el 22 de juliol de 1922», [Drame en trois actes de Maurice Maeterlinck. Première à Barcelone au théâtre Cirera le 22 juillet 1922], traduction de Josep M. Jordà et Francesc Pujols, La Escena Catalana, 12-81922, Ve Année (2 époque), no 111.

La vida de les abelles, version catalane de Cecili Gasòliba, Barcelone, Llibreria Catalònia, (Biblioteca Literària, 115), 1929. Compte-rendu et étude du P. Miquel d'Esplugues. "La vida de les abelles" de Maeterlinck». Criterion. Revista trimestral de filosofia, fasc. 17 (avril-juin de 1929), 127-142.

El despertar del alma [Le reveil de l'âme], sans nom de traducteur, Barcelone, Agencia Editorial Presa; Madrid, Gregorio Pueyo, s. d. [192?]

La intel-ligència de les flors, traduction de Jaume dels Domenys [pseudonyme d'Alfons

Maseras], Barcelone, Llibreria Catalònia, (Biblioteca Literària, 122), s. a. [1930]

Debussy Claude, Pel-leas i Melisanda [Pelléas et Mélisande], «Drama líric en cinc actes i 12 quadres. Poema de Maurici Maeterlinck. Estrenat a París el 1902 i representat a Barcelona per primera vegada al teatre Tívoli l'11 d'octubre de 1919». [Drame lyrique en cinq actes et 12 tableaux. Poème de Maurice Maeterlinck. Première à Paris en 1902 et représenté à Barcelone pour la première fois au théâtre Tivoli le 11 octobre 1919]. Traduction adaptée à la musique de Joaquim Pena, Barcelone, Arts Gràfiques S.A. Successors d'Henrich i Cia, 1930. 
L'ocell blau. Conte de fades, en sis actes i dotze decorats [L'oiseau bleu: Conte de fées en six actes et douze tableaux]. sans nom de traducteur. Photocopie d'un texte mécanographié sans date (non antérieur à 1911) conservé à la Bibliothèque de Catalogne (référence 2006-4-6365).

[Non publiée, mais annoncée] Peleàs i Melisenda. Col. Biblioteca Femina (que en homenatge a la dona catalana publica en J. Massó Ventós). [Ca. 1920]. [Dans cette collection, la publication de cette œuvre de Maeterlinck est annoncée pour le numéro 5; en fait, seul le premier ( Les Nits, de Musset), a été publié en toute certitude]

[Non publiée, mais représentée] Maria Magdalena. Traduction de Carles Capdevila. Première au Teatre Romea de Barcelone le 8 mars 1921.

[Non publiée, mais représentée] Sor Beatriz. Légende dramatique adaptée à la scène lyrique par Ventura Gassol. Musique d'Antonio Marqués. Gran Teatre del Liceu de Barcelone. Première le 20 décembre 1924.

"Prólogo» à Pierre Devoluy \& Pierre Borel, La Costa Azul: Niza y La Riviera, traduction espagnole d'Enrique de Leguina, Barcelone, Edita, s. a. [1927].

Junoy Josep Maria, "La idea de la muerte en la obra de Mauricio Maeterlinck», $L a$ Vanguardia, 19-12-1934. 